\title{
Positronium formation from valence and inner shells in noble gas atoms
}

\author{
L. J. M. Dunlop, G. F. Gribakin* \\ Department of Applied Mathematics and Theoretical Physics, Queen's University \\ Belfast, Belfast BT7 1NN, Northern Ireland, UK
}

\begin{abstract}
When recent experimental positronium (Ps) formation cross sections in noble gases have been compared with the most up-to date theoretical studies, the agreement is qualitative, but not quantitative. In this paper we re-examine this process and show that at low energies Ps formation must be treated nonperturbatively. We also look at Ps formation with inner shell electrons.
\end{abstract}

Key words: Positron scattering, Positronium formation, inner-shell ionization PACS: 34.85.+x, 36.10.Dr, 32.80.Hd

\section{Introduction}

Positronium (Ps) represents a bound state between a positron and an electron. It is formed in positron-atom collisions,

$$
A+e^{+} \longrightarrow A^{+}+\mathrm{Ps}
$$

when the positron energy, $\varepsilon=k^{2} / 2$, is above the Ps formation threshold,

$$
\varepsilon>\left|\varepsilon_{n}\right|-\left|E_{1 s}\right|
$$

where $\varepsilon_{n}$ is the energy of the bound electron atomic orbital $n, E_{1 s} \approx-6.8 \mathrm{eV}$ is the energy of the ground-state Ps, and $k$ is the incident positron momentum (atomic units are used throughout).

\footnotetext{
* Corresponding author

Email addresses: 1.dunlop@qub.ac.uk (L. J. M. Dunlop), g.gribakin@qub.ac.uk (G. F. Gribakin).
} 
Recently positronium formation in $\mathrm{Ne}, \mathrm{Ar}, \mathrm{Kr}$ and Xe has been determined by two experimental groups $[1,2]$. The two sets of data are in fairly good agreement, especially at lower energies. However, recent distorted-wave Born approximation (DWBA) calculations [3] overestimate the cross sections by a large factor, ranging from 1.6 in Ne to 3 in Xe, while the overall energy dependence of the DWBA cross sections is in reasonable accord with experiment. This is in contrast with earlier coupled-static calculations [4], which yield better magnitudes of the cross section maxima, but disagree on the energy dependence.

In this paper we perform 1st-order and all-order calculations of Ps formation from valence and subvalence subshells. Our consideration is restricted to Ps formation in the ground-state. Noble gas atoms have tightly bound electrons, making excited-state Ps formation much less probable (see, e.g., [3]). We argue that a structure observed at energies beyond the main cross section maximum (described as a shoulder, or in some cases seen as a secondary peak $[1,5]$ ) is most likely related to Ps formation by the subvalence $n s$ electrons. We also consider Ps formation from inner shells. It produces inner-shell vacancies and can be important for positron-annihilation-induced Auger-electron spectroscopy [6].

\section{1st-order approximation}

\subsection{Ps formation amplitude and cross section}

Using 1st-order many-body perturbation theory, and neglecting the interaction between the outgoing Ps and residual ion, the Ps-formation amplitude can be written as [7]

$$
\left\langle\widetilde{\Psi}_{1 s, \mathbf{K}}|V| n, \varepsilon\right\rangle=\int \widetilde{\Psi}_{1 s, \mathbf{K}}^{*}\left(\mathbf{r}_{1}, \mathbf{r}_{2}\right)\left(-\frac{1}{\left|\mathbf{r}_{1}-\mathbf{r}_{2}\right|}\right) \psi_{n}\left(\mathbf{r}_{2}\right) \varphi_{\varepsilon}\left(\mathbf{r}_{1}\right) d \mathbf{r}_{1} d \mathbf{r}_{2}
$$

where $\varphi_{\varepsilon}$ is the incident positron wavefunction, $\psi_{n}$ is the Hartree-Fock wave-

function of the initial electron state ("hole"), and $\widetilde{\Psi}_{1 s, \mathbf{K}}$ is obtained from the wavefunction of the ground-state Ps with momentum $\mathbf{K}$,

$$
\Psi_{1 s, \mathbf{K}}\left(\mathbf{r}_{1}, \mathbf{r}_{2}\right)=e^{i \mathbf{K} \cdot\left(\mathbf{r}_{1}+\mathbf{r}_{2}\right) / 2} \phi_{1 s}\left(\mathbf{r}_{1}-\mathbf{r}_{2}\right),
$$


by orthogonalising it to all electron orbitals $n^{\prime}$ occupied in the target ground state,

$$
\widetilde{\Psi}_{1 s, \mathbf{K}}=\left(1-\sum_{n^{\prime}}\left|n^{\prime}\right\rangle\left\langle n^{\prime}\right|\right) \Psi_{1 s, \mathbf{K}} .
$$

The positron wavefunction is calculated in the field of the ground state atom described in the Hartree-Fock approximation. The Ps center-of-mass motion is described by a plane wave. The Ps formation cross section is found by integration over the directions of $\mathbf{K}$,

$$
\sigma_{\mathrm{Ps}}=\frac{M K}{4 \pi^{2} k} \int\left|\left\langle\widetilde{\Psi}_{1 s, \mathbf{K}}|V| n, \varepsilon\right\rangle\right|^{2} d \Omega_{\mathbf{K}}
$$

where $M=2$ and $K=\left[2 M\left(\varepsilon-\left|\varepsilon_{n}\right|+\left|E_{1 s}\right|\right)\right]^{1 / 2}$ are the Ps mass and momentum, and $\phi_{\varepsilon}(\mathbf{r}) \sim e^{i \mathbf{k} \cdot \mathbf{r}}$ normalization is assumed. The approximation (3)-(6) is equivalent to DWBA for a rearrangement collision.

A numerical calculation of the amplitude and cross section was performed by expanding the Ps wavefunction in electron and positron spherical harmonics with respect to the nucleus. Integration over the angular variables was done analytically, while the radial integrals are calculated numerically (see [7] for some details). To ensure accurate positions of the Ps formation thresholds, experimental ionization energies $\left|\varepsilon_{n}\right|$, rather than the Hartree-Fock values, were used in the calculations. Detailed below are the Ps-formation cross sections for neon, argon, krypton and xenon, calculated using the 1st-order approximation described above.

\subsection{Partial-wave contributions}

The cross sections are found by summing over the positron partial waves from $l=0$ to 10 . Figure 1 shows the partial wave contributions for the $3 p$ subshell of argon. Note that the $p, d, f$ and $g$ waves have the largest individual cross sections and make up most of the cross section peak. The contributions of higher partial waves are suppressed by the centrifugal barrier, preventing the close encounters which lead to Ps formation. The small contribution of the $s$-wave is due to it being spherically symmetric, making it harder for the positron to bind and move away with an electron. This is true for all the noble gases. The exceptionally small $s$-wave contribution to Ps formation was noticed earlier for hydrogen and helium (see, e.g., $[8,9,10]$ ) and explained by the hidden-crossing method [11]. 


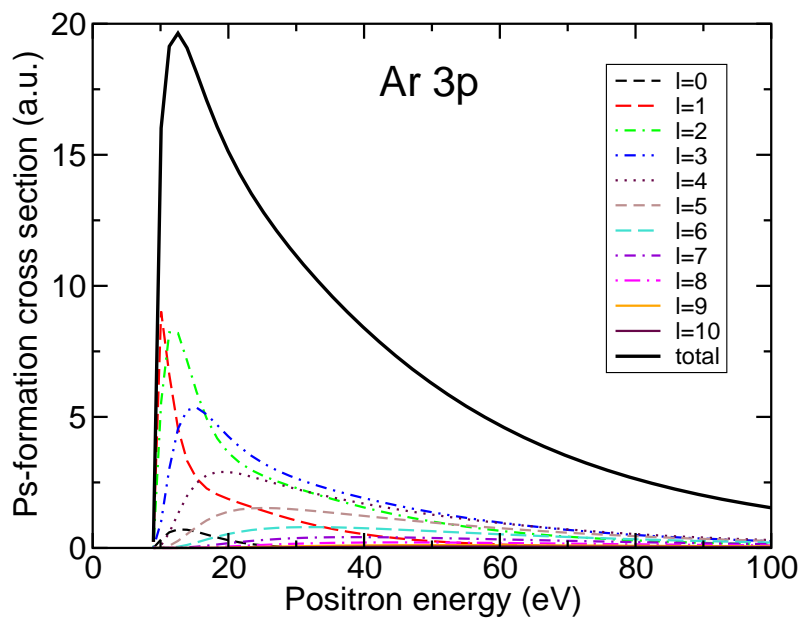

Fig. 1. Partial wave contributions to the total Ps formation cross section for the $3 p$ subshell of argon. Various thin curves are the contributions of $l=0-10$, while the solid thick curve is the total cross section.

\subsection{Comparison with experiment}

Figure 2 shows the Ps-formation cross sections for the valence $n p$ and subvalence $n s$ orbitals together with their sum, for Ne, Ar, Kr and Xe. The present results for the $n p$ subshell practically coincide with the $\operatorname{Ps}(1 s)$ formation cross section from DWBA [3]. The calculations are compared with the experimental data obtained with a cold, trap-based positron beam [2], and with the cross section found by subtracting the direct ionization cross section from the total ionization cross section [1]. Moving from Ne to Xe, the calculations increasingly overestimate the measured cross section near the maximum.

For Ne, Ar and Xe experiment and theory converge at higher energies, while in $\mathrm{Kr}$ the discrepancy persists. Such convergence should be expected from a theory point of view. Indeed, at higher positron energies the dominant contribution to the amplitude (3) and cross section (6) comes from higher partial waves, for which the plane-wave description of the Ps motion is more accurate. At the same time, the contributions of individual partial waves to the amplitude become small. This means that higher-order corrections neglected by the 1st-order theory may not be important (see below). Thus, we cannot offer an explanation for the divergence between theory and experiment in Kr.

\subsection{Inner-shell Ps formation}

Ps-formation thresholds for the inner shells lie at much higher energies, e.g., at 242 and $320 \mathrm{eV}$ for the $2 p$ and $2 s$ orbitals in Ar. As a result, the incident positron wavefunction oscillates rapidly, reducing the magnitude of the am- 

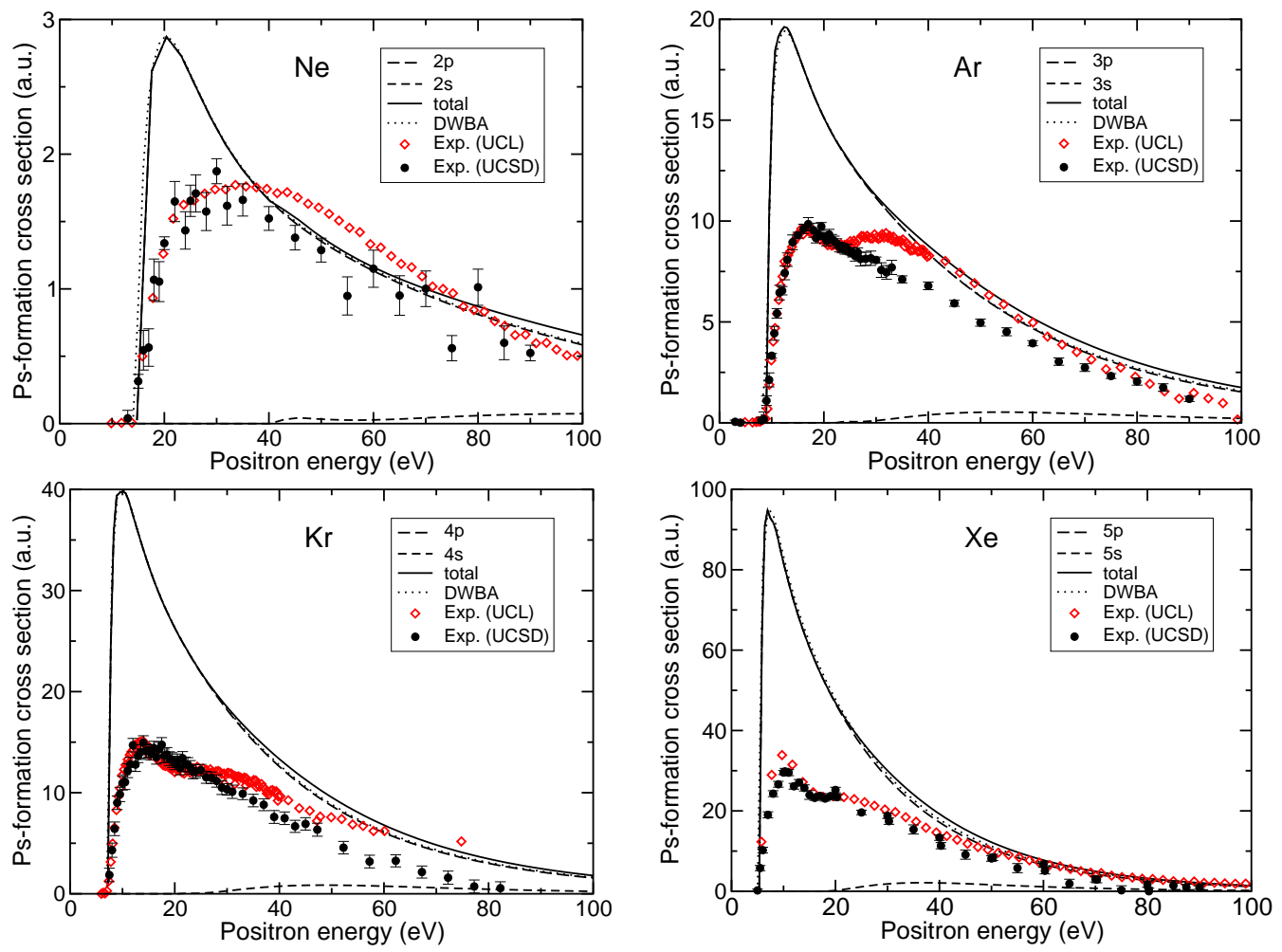

Fig. 2. Comparison of the 1st-order Ps-formation cross sections for $\mathrm{Ne}, \mathrm{Ar}, \mathrm{Kr}$ and Xe with experiment. Calculations: long-dashed curve, contribution of the $n p$ subshell; short-dashed curve, contribution of the $n s$ subshell; solid curve, total cross section; dotted curve, DWBA [3] for the $n p$ subshell. Experiment: solid circles, University of California at San Diego (UCSD) [2]; open diamonds, University College London (UCL) [1].

plitude (3). Ps formation from inner shells is additionally suppressed by the positron repulsion from the nucleus. The Ps-formation cross sections for Ne, Ar, Kr and Xe are shown in Figure 3. It can be noted that significantly higher energies are required to produce positronium for the lighter noble gases (with the more tightly bound electrons).

In general, the Ps-formation cross sections for the inner-shell electrons are quite small. Thus, in neon, the valence shell contribution still dominates, even at positron energies of $1600 \mathrm{eV}$. In argon, it can be seen that up to $500 \mathrm{eV}$, the valence contribution dominates the cross section. However, above this energy all subshells contribute approximately equally to the total cross section, and as the energy increases further, the $2 p$ subshell contributes the most. In krypton, the $3 d$ subshell contributes most to the Ps-formation cross section, as this subshell is relatively far away from the nucleus. This is also true for xenon, where the $4 d$ subshell dominates the cross section. Note also that in $\mathrm{Kr}$ and especially Xe the inner-shell cross sections are much larger than those of Ne and even Ar, with their Ps formation threshold values nearly ten times smaller than that of $1 s$ in $\mathrm{Ne}$. 

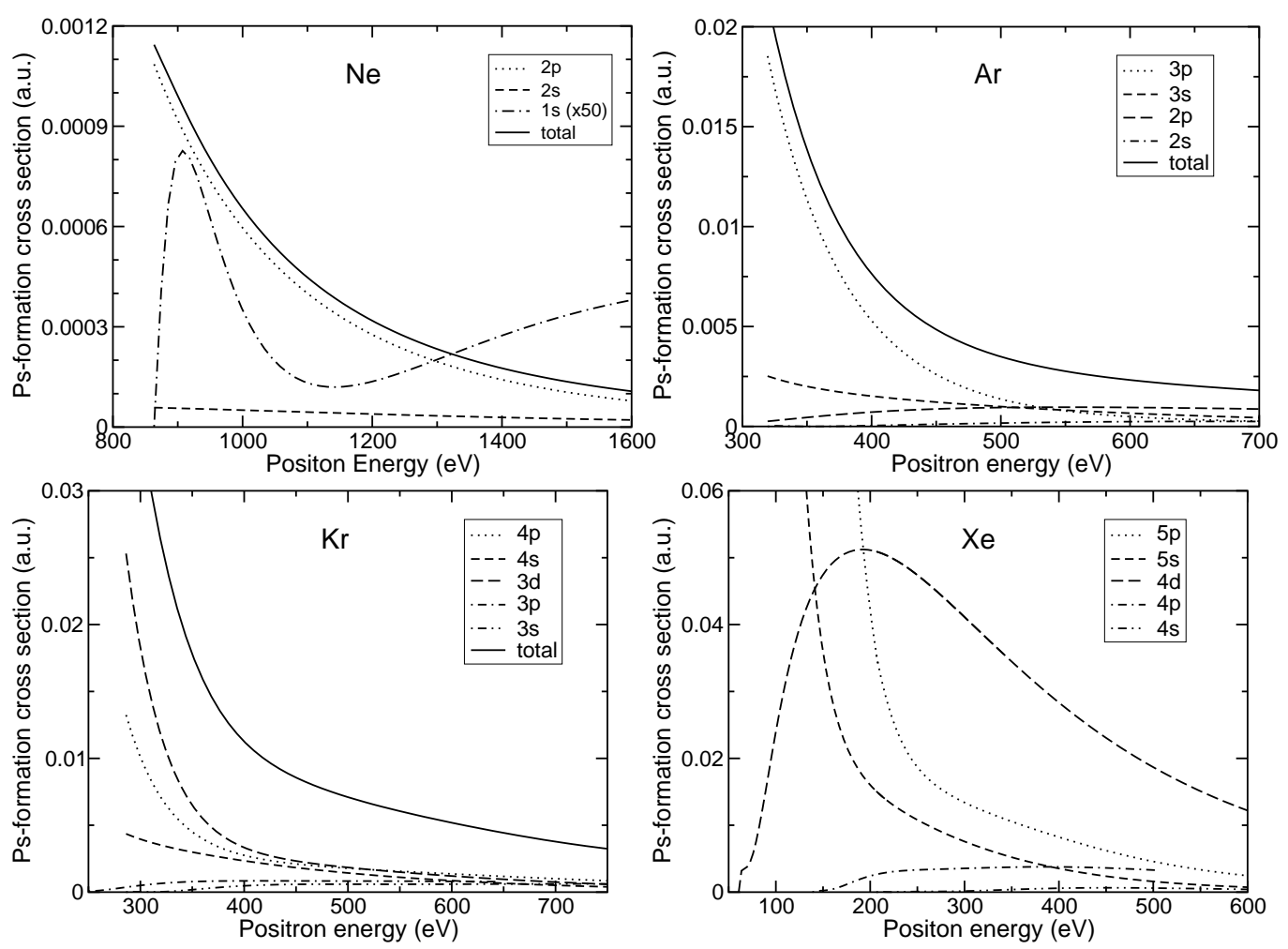

Fig. 3. Ps-formation cross sections for inner and valence subshells of $\mathrm{Ne}, \mathrm{Ar}, \mathrm{Kr}$ and Xe.

\section{Ps formation: nonperturbative approach}

\subsection{Check of unitarity}

As seen from Figure 2, the Ps formation cross sections increase dramatically from Ne to Xe. This increase is matched by a growing discrepancy between the 1st-order results and experiment. This suggests that as the cross sections become larger, the lowest-order perturbation theory treatment becomes increasingly inaccurate. Indeed, it turns out that in the 1st-order approximation, equations $(3)-(6)$, the lower partial-wave contributions $(l=0-3)$ which dominate near the cross section maximum, become close to and even violate (for $\mathrm{Kr}$ and $\mathrm{Xe}$ ) the unitarity limit for inelastic processes, $\sigma_{\mathrm{Ps}}^{(l)} \leq \pi(2 l+1) / k^{2}$. A comparison with the unitarity limit is presented in Figure 4 for the $s$ and $p$ partial waves in $\mathrm{Ar}, \mathrm{Kr}$ and Xe.

Physically, unitarity ensures that the amount of Ps formed in positron-atom collisions cannot be greater than the number of positrons going in. Any inelastic cross section above this limit is physically impossible. The fact that our 1st-order results (and the analogous DWBA cross sections of Ref. [3]) are close to, or exceed the unitarity limit means that Ps formation is a strong process which cannot be treated perturbatively. Hence, in addition to the 1st- 

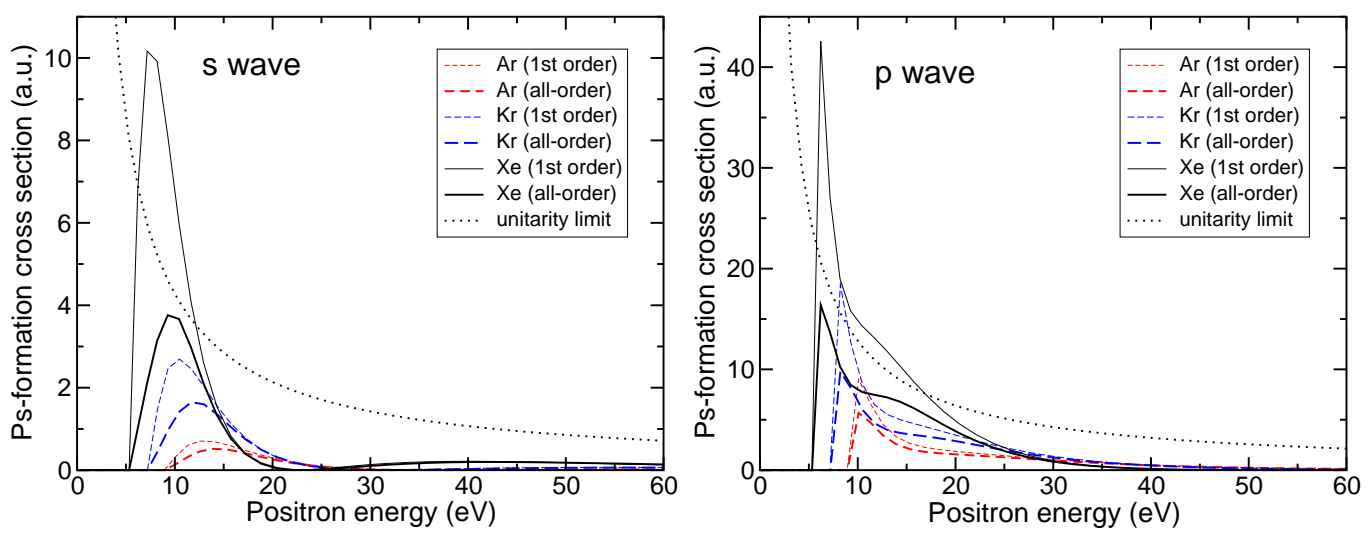

Fig. 4. Comparison of the $s$ - and $p$-wave partial Ps formation cross sections for Ar, $\mathrm{Kr}$ and Xe with the unitarity limit. Thin curves, 1st-order approximation; thick curves, all-order approximation; dotted curves, unitarity limit, $\pi(2 l+1) / k^{2}$.

order Ps-formation amplitude (3), one must include higher-order contributions which account for the decay of $A^{+}$Ps back into the $e^{+}+A$ channel, followed by Ps formation, etc. In other words, the effect of Ps formation on the incident positron must be taken into account. We do this by means of an all-order approach outlined in the next section.

\subsection{All-order approximation}

The effect of Ps formation on the positron can be described by the Psformation contribution to the positron-atom correlation potential, defined by its matrix elements [7],

$$
\left\langle\varepsilon^{\prime}\left|\Sigma_{E}^{(\mathrm{Ps})}\right| \varepsilon\right\rangle=\int \frac{\left\langle\varepsilon^{\prime}, n|V| \widetilde{\Psi}_{1 s, \mathbf{K}}\right\rangle\left\langle\widetilde{\Psi}_{1 s, \mathbf{K}}|V| n, \varepsilon\right\rangle}{E+\varepsilon_{n}-E_{1 s}-K^{2} / 4+i 0} \frac{d^{3} K}{(2 \pi)^{3}}
$$

where $\left\langle\widetilde{\Psi}_{1 s, \mathbf{K}}|V| n, \varepsilon\right\rangle$ is the amplitude (3), $E_{1 s}+K^{2} / 4$ in the denominator is the Ps energy, and the integral is over all Ps momenta $\mathbf{K}$. Note that in this section we use positron states with a given angular momentum, i.e., spherical waves, $\varphi_{\varepsilon}(\mathbf{r})=r^{-1} Y_{l m}(\Omega) P_{\varepsilon l}(r)$, with the radial wavefunctions normalized by $P_{\varepsilon l}(r) \sim(\pi k)^{-1 / 2} \sin \left(k r-\frac{1}{2} \pi l+\delta_{l}^{\mathrm{HF}}\right)$, where $\delta_{l}^{\mathrm{HF}}$ is the positron phaseshift in the static field of the Hartree-Fock atom. In this case the correlation potential (7) is determined separately for each positron partial wave.

Below the Ps-formation threshold $\left\langle\varepsilon^{\prime}\left|\Sigma_{E}^{(\mathrm{Ps})}\right| \varepsilon\right\rangle$ is real. Above the Ps-formation threshold, for $E>\left|\varepsilon_{n}\right|-\left|E_{1 s}\right|$, the correlation potential acquires an imaginary part. This gives rise to "absorption" of the positron flux, which is being redirected into the Ps formation channel. In fact, the 1st-order Ps formation 
cross section (6) is proportional to the imaginary part of $\left\langle\varepsilon\left|\Sigma_{\varepsilon}^{(\mathrm{Ps})}\right| \varepsilon\right\rangle$,

$$
\operatorname{Im}\left\langle\varepsilon\left|\Sigma_{\varepsilon}^{(\mathrm{Ps})}\right| \varepsilon\right\rangle=-\pi \frac{M K}{(2 \pi)^{3}} \int\left|\left\langle\tilde{\Psi}_{1 s, \mathbf{K}}|V| n \varepsilon\right\rangle\right|^{2} d \Omega_{\mathbf{K}}
$$

where $K=2\left(\varepsilon-\left|\varepsilon_{n}\right|+\left|E_{1 s}\right|\right)^{1 / 2}$. Because of the different normalization of the positron states adopted in Secs. 2 and 3.2, caution is required when using equations (6) and (8) to relate $\sigma_{\mathrm{Ps}}$ to $\operatorname{Im}\left\langle\varepsilon\left|\Sigma_{\varepsilon}^{(\mathrm{Ps})}\right| \varepsilon\right\rangle$ (such relation is derived below).

A nonperturbative ("all-order") calculation of Ps formation is done by solving an integral equation for the matrix elements of $\tilde{\Sigma}_{E}$ (see, e.g., [12]),

$$
\left\langle\varepsilon^{\prime}\left|\tilde{\Sigma}_{E}\right| \varepsilon\right\rangle=\left\langle\varepsilon^{\prime}\left|\Sigma_{E}^{(\mathrm{Ps})}\right| \varepsilon\right\rangle+\int \frac{\left\langle\varepsilon^{\prime}\left|\tilde{\Sigma}_{E}\right| \varepsilon^{\prime \prime}\right\rangle\left\langle\varepsilon^{\prime \prime}\left|\Sigma_{E}^{(\mathrm{Ps})}\right| \varepsilon\right\rangle}{E-\varepsilon^{\prime \prime}} d \varepsilon^{\prime \prime}
$$

The positron scattering phaseshift is then obtained as

$$
\delta_{l} \equiv \delta_{l}^{\prime}+i \delta_{l}^{\prime \prime}=\delta_{l}^{\mathrm{HF}}+\Delta \delta_{l}
$$

with

$$
\tan \Delta \delta_{l}=-\pi\left\langle\varepsilon\left|\tilde{\Sigma}_{\varepsilon}\right| \varepsilon\right\rangle
$$

where $\Delta \delta_{l}$ is the additional phaseshift due to the correlation potential. For energies $E$ above the Ps formation threshold, the correlation potential, $\Sigma_{E}^{(\mathrm{Ps})}$, is complex, and the phaseshift has a nonzero imaginary part, $\delta_{l}^{\prime \prime}>0$. The Psformation cross section is then obtained from $\delta_{l}^{\prime \prime}$ by summing over the partial waves,

$$
\sigma_{\mathrm{Ps}}=\frac{\pi}{k^{2}} \sum_{l=0}^{\infty}(2 l+1)\left(1-e^{-4 \delta_{l}^{\prime \prime}}\right) .
$$

If we assume that Ps formation is a weak process, then $\left\langle\varepsilon\left|\Sigma_{E}^{(\mathrm{Ps})}\right| \varepsilon\right\rangle$ is small, and we have $\left\langle\varepsilon\left|\tilde{\Sigma}_{E}\right| \varepsilon\right\rangle \approx\left\langle\varepsilon\left|\Sigma_{E}^{(\mathrm{Ps})}\right| \varepsilon\right\rangle, \Delta \delta_{l} \approx-\pi\left\langle\varepsilon\left|\tilde{\Sigma}_{\varepsilon}\right| \varepsilon\right\rangle$, and $\delta_{l}^{\prime \prime} \approx-\pi \operatorname{Im}\left\langle\varepsilon\left|\Sigma_{\varepsilon}^{(\mathrm{Ps})}\right| \varepsilon\right\rangle$. Using $\delta_{l}^{\prime \prime} \ll 1$, we then have from equation (12):

$$
\sigma_{\mathrm{Ps}}^{(l)} \approx-\frac{4 \pi^{2}}{k^{2}}(2 l+1) \operatorname{Im}\left\langle\varepsilon\left|\Sigma^{(\mathrm{Ps})}\right| \varepsilon\right\rangle .
$$

The right-hand side of this equation is equivalent to the 1st-order approximation examined earlier in this paper. 


\subsection{Total Ps-formation cross sections}

Figure 5 shows the all-order and 1st-order cross sections for $\mathrm{Ne}, \mathrm{Ar}, \mathrm{Kr}$ and Xe along with the two sets of experimental data. As expected, the difference between the all-order and 1st-order results is greater for atoms where the Ps formation cross section is large, i.e., for $\mathrm{Kr}$ and Xe. The all-order approximation has reduced the cross section maxima for all atoms, but still not significantly enough to match the experiment. However, theory and experiment are in better agreement at higher positron energies. The situation looks especially encouraging in Xe, where both experiments and theory are close above $40 \mathrm{eV}$. Note that for $\mathrm{Kr}$ and Xe, the all-order and 1st-order cross sections are markedly different even at the higher-energy part of the scale. This emphasizes the need for nonperturbative treatment of Ps formation.
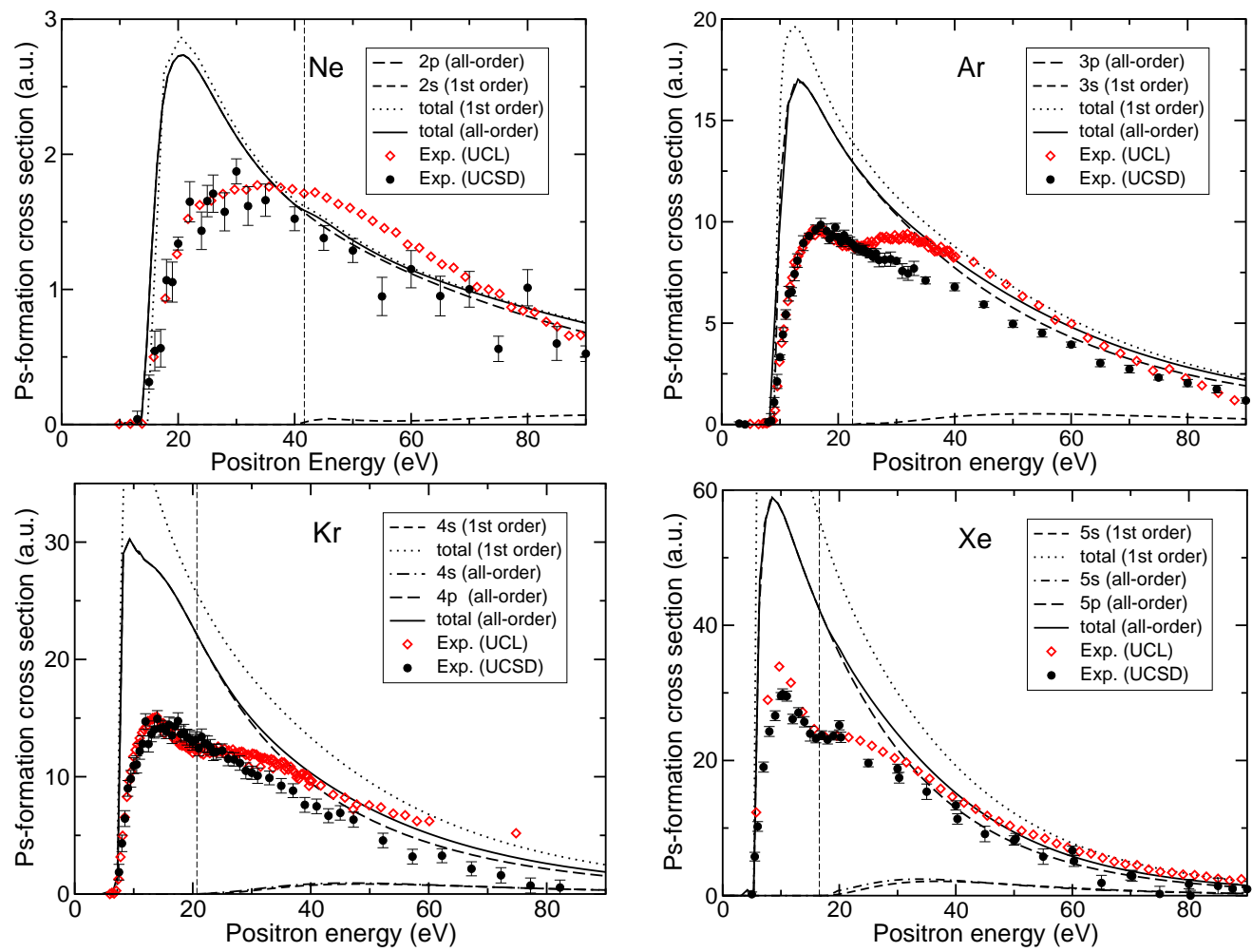

Fig. 5. Comparison of the calculated all-order and 1st-order Ps-formation cross sections for Ne, Ar, Kr and Xe with experiment. Calculations: dotted curve, total (1st-order); short-dashed curve, $n s$ subshell (1st order); long-dashed curve, $n p$ subshell (all-order); chain curve, ns subshell (all-order); solid curve, total cross section (all-order). Experiment: solid circles, UCSD [2]; open diamonds, UCL [1]. Vertical dashed lines indicate Ps-formation thresholds for the subvalence $n s$ electrons.

Regarding the contribution of the subvalence $n s$ subshell, the corresponding Ps-formation thresholds for Ne, Ar, Kr and Xe are 41.67, 22.47, 20.71 and $16.59 \mathrm{eV}$, respectively ${ }^{1}$. The all-order calculation was not performed for $\mathrm{Ne}$

$\overline{1}$ Note that the $n s$ thresholds indicated in figures 5-8 of Ref. [1] are ns ionization 
and Ar, where we expected its effect to be insignificant. In fact, the $2 s$-subshell contribution to the Ps-formation cross section in Ne is very small. Experimental data in Ne also do not reveal any clear features that could be related to the opening of a new channel at $\varepsilon \approx 42 \mathrm{eV}$. The Ps formation from the $3 \mathrm{~s}$ subshell in $\mathrm{Ar}$ is relatively more important. The opening of this channel at about $22.5 \mathrm{eV}$ coincides with the onset of the second peak in the UCL data [1]. It also marks the start of a weak shoulder-like structure in the UCSD data [2], where earlier experiments [5] showed a much more prominent feature. The reason for the discrepancy between different experimental observations is at present unclear, but we suggest that the origin of this structure is most likely related to the opening of the $3 s$ Ps-formation channel.

Using the all-order approach makes a much greater difference in krypton and xenon. The valence $n p$ contribution is greatly reduced in the energy range around the maximum. In contrast, the subvalence $n s$ contribution is increased in the all-order approach. The onset of the Ps-formation cross section from the $4 s$ orbital in $\mathrm{Kr}$ is very smooth. The UCSD data for Kr do not show any feature just above $20 \mathrm{eV}$, while the UCL data possess a clear shoulder, whose onset is more rapid than that predicted by the theory. At higher energies the two sets of experimental data diverge, with our all-order results being inbetween. Given that the theory is expected to be more reliable here than at lower energies, we suggest that this discrepancy points to a need for further experimental studies.

Of the four atoms examined, Xe has the largest Ps formation cross section by the subvalence electrons. Its contribution results in a change of slope of the calculated total cross section. For Xe both sets of experimental data show a clear shoulder-like structure, whose onset is close to the $5 s$ Ps-formation threshold. The overall size of the shoulder is comparable with the calculated $5 s$ Ps formation cross section (chain curve in Figure 5). At higher energies there is good agreement between the two experiments and the calculated cross section.

One may speculate that in a better calculation, the Ps-formation cross section from the valence $n p$ orbital will be suppressed around its maximum and at the energies below $40 \mathrm{eV}$. Then, even if the $n s$ Ps-formation cross sections remain close to the present estimates, their contribution to the total will be more noticeable.

Finally, an alternative explanation of the shoulder/secondary-peak structure discussed, e.g., in Ref. [1], is that it is caused by Ps formation in excited states. According to the DWBA calculations of Gilmore et al. [3], this contribution is not negligible (though small). However, the thresholds for excited-state Ps formation lie much lower than the energies where the structures are observed,

thresholds, rather than the Ps-formation thresholds. 
making its importance for these structures questionable.

\section{Conclusions}

A comparison of the 1st-order and all-order results shows that as Ps formation is strong, it cannot be treated perturbatively. Going beyond the 1st-order approximation reduces the cross sections, especially at low energies. However, below $40 \mathrm{eV}$ the calculated cross sections are still higher than experimental values. Above this energy theory and experiment generally converge. In particular, in Xe we observe good agreement between the calculations and experimental data from the UCL and UCSD groups.

There are two reasons for the discrepancy between theory and experiment at low energies. First, the motion of Ps in our calculations is described by a plane wave. The electron part of the Ps wavefunction is orthogonalized to the target electron orbitals. This manifestation of the Pauli principle to some extent accounts for the interaction between the Ps and the final-state ion. On the other hand, the positron repulsion from the nucleus in the final Ps state is completely neglected. This repulsion is especially important for the lower positron partial waves. Its neglect is probably the main reason for the overestimation of the Ps-formation cross section maxima by the present method.

Secondly, all open channels, i.e., elastic scattering, Ps formation and direct ionization must be included simultaneously. Above the atomic ionization threshold all of these channels compete for the positron flux. This effect can be accounted for by the correlation potential method described in Sec. 3.2, by adding the lowest 2 nd-order contribution to $\Sigma_{E}$ (see, e.g., [13]). However, to be able to extract the Ps formation cross section from such a calculation, the formalism of Sec. 3.2 must be extended. In the present form the imaginary part of the phaseshift allows one to find only the total reaction cross section.

One may expect that both of these effects will make the Ps formation cross section smaller, and bring it into a close agreement with experiment. By further including Ps formation from the inner valence subshell and Ps formation in excited states, one should achieve a complete description of the Ps formation process, including any secondary structures. 


\section{Acknowledgements}

We are grateful to G. Laricchia and D. Murtagh (UCL), C. Surko and J. Marler (San Diego) and H. R. J. Walters and S. Gilmore (Belfast) for providing us with their data in table form and useful discussions. LJMD acknowledges DEL (Northern Ireland) for support in the form of a $\mathrm{PhD}$ studentship.

\section{References}

[1] G. Laricchia, P. Van Reeth, M. Szłuińska and J. Moxom J. Phys. B 35 (2002) 2525.

[2] J. P. Marler, J. P. Sullivan and C. M. Surko, Phys. Rev. A 71 (2005) 022701.

[3] S. Gilmore, J. E. Blackwood and H. R. J. Walters, Nucl. Instrum. Methods B 221 (2004) 129.

[4] M. T. McAlinden and H. R. J. Walters, Hyperfine Interactions 73 (1992) 65.

[5] M. Charlton, G. Clark, T. C. Griffith, and G. R. Heyland, J. Phys. B 16 (1983) L465.

[6] T. Ohdaira, R. Suzuki, Y. Kobayashi, T. Akahane and L. Dai, Appl. Surface Science 194 (2002) 291.

[7] V. A. Dzuba, V. V. Flambaum, G. F. Gribakin and W. A. King, J. Phys. B 29 (1996) 3151.

[8] J. W. Humberston, Can. J. Phys. 60 (1982) 591.

[9] P. Van Reeth, J. W. Humberston, J. Phys. B 28 (1995) L511.

[10] P. Van Reeth, J. W. Humberston, J. Phys. 30 (1997) L95.

[11] S. J. Ward, J. H. Macek and S. Yu. Ovchinnikov, Phys. Rev. A 59 (1999) 4418.

[12] M. Ya. Amusia, N. A. Cherepkov, L. V. Chernysheva, D. M. Davidović, and V. Radojević, Phys. Rev. A 25 (1982) 219.

[13] G. F. Gribakin and W. A. King, Can. J. Phys. 74 (1996) 449. 